\title{
Changes in the Peripheral Treg Cell Proportion and Immune Function in Hepatocellular Carcinoma Patients After Transarterial Chemoembolization with Gelatin Sponge Microparticles
}

\author{
Zhizhong Ren ${ }^{1}$, Yuanxun Yue ${ }^{2}$, Yuewei Zhang ${ }^{1}$, Xin $\operatorname{Lin}^{3}$, Xueqiang Zhao ${ }^{4}$, Ying Liu ${ }^{1}$, \\ Xiaowei Yang ${ }^{1}$, and Xin Huang ${ }^{1}$ \\ ${ }^{1}$ Tsinghua University Affiliated Beijing Tsinghua Changgung Hospital \\ ${ }^{2} \mathrm{Lu}$ He Hospital, Capital Medical University \\ ${ }^{3}$ Institute for ImmunologySchool for MedicineTsinghua University \\ ${ }^{4}$ Tsinghua Univ
}

May 5, 2020

\begin{abstract}
Objective: Transarterial chemoembolization (TACE) stands for an ideal therapy for patients with intermediate stage HCC. This study was carried out to observe the effect of gelatin sponge microparticles-transarterial chemoembolization (GSMs-TACE) on the immune function of patients with liver cancer by detecting the proportion of Treg cells in the peripheral blood of HCC patients before and after the treatment of GSMs-TACE, and to determine whether GSMs-TACE has a positive regulatory effect on the immune function of patients with liver cancer. Methods: 28 HCC patients treated with GSMs-TACE were enrolled. Flow cytometry was used to determine the Treg cell proportion in peripheral blood in all patients with HCC 1 day before GSMs-TACE, 10 days and 30 days after GSMs-TACE, respectively. Results: The Tregs cell proportion in peripheral blood of primary HCC patients was significantly higher than that of the healthy controls and was associated with various clinical indicators of HCC. The Treg cell proportion in the peripheral blood of patients with BCLC stage C liver cancer was higher than that of stage B patients; The Treg cell proportion at 10 days postoperatively was $8.85 \pm 1.23 \%$, which was significantly lower than that before the GSMs-TACE. The Treg cell proportion at 30 days postoperatively was $10.27 \pm 1.28 \%$, which remained in the low range. Conclusion: These results indicated that GSMs-TACE could exert a positive regulatory effect on the anticancer immune function of HCC patients, which may be used in combination with immune adjuvant therapies to enhance the treatment efficacy of HCC.
\end{abstract}

\section{Introduction}

Hepatocellular carcinoma (HCC) is the most common malignancy worldwide. Currently, liver transplantation and surgical resection remain the primary choice for HCC, but the overall survival of HCC patients is still depressing mainly due to local recurrence, distant metastasis, treatment resistance, and the lack of early diagnosis [1-3]. Transarterial chemoembolization (TACE) is one of the first-line treatment choices for HCC patients with specific clinical characteristics, such as stage B according to Barcelona Clinic Liver Cancer (BCLC)[4]. The transcatheter delivery of both chemotherapeutic agents and embolizing agents contributes to a dual effect of cytotoxicity and ischemia in tumor tissues [4]. Currently, there are mang TACE modalities in clinical practice, such as conventional TACE (cTACE), GSMs-TACE and DEB-TACE. [4].

As the representative of absorbable particles, gelatn sponge microparticles are characterized by chemical cross-linking and physical adsorption since they can be absorbed 7-15 days after arterial embolization. Since 2009, satisfactory efficacy and safety of GSMs-TACE in the treatment of HCC patients at stage B and C 
(BCLC classification) with a diameter of $150 \mu \mathrm{m}-350 \mu \mathrm{m} / 350 \mu \mathrm{m}-560 \mu \mathrm{m}$ GSMs have been achieved in our team $[5,6]$. During GSMs-TACE procedures, under the premise of good liver function, the standard of terminating embolization is that the tumor blood supply artery is completely embolized. Compared with cTACE, the application of TACE combined with different microparticles in the treatment of liver cancer can lead to more significant tumor necrosis, especially the huge liver tumors.

Since the identification of tumor antigens, various approaches manipulating the immune system have been developed for cancer therapies [7]. However, not all patients respond to immunotherapies, and one of the major obstacles is the formation of immunosuppressive tumor microenvironment which is filled with immunosuppressive cells such as myeloid-derived suppressor cells and regulatory T cells [8]. Treg cells are a T-lymphocyte subset and help maintain immune homeostasis by controlling abnormal/excessive immune responses. Studies have found that Treg cells are also involved in development and progression of tumors via acting as s suppressor of effective antitumor immunity [7, 9]. High infiltration by Treg cells into TME was observed in various types of tumors including HCC and was found to be correlated with poor prognosis [8-10]. Therefore, strategies to reduce Treg cells and control the functions of Treg cells would be potentially effective anticancer therapies.

Many studies have confirmed the efficacy and safety of TACE procedures in treating HCC, and it was considered that the effects were mainly attributed to the blood supply blockade and the cytotoxic effects $[11,12]$. Except for the tumor necrosis, it was also observed that the lesions of extrahepatic metastasis were reduced or even disappeared as described in our clinical practice. Considering the close relationship between tumor metastasis and the immunosuppressive TME, these findings prompted us wonder if the effect of TACE was also partly attributed to that the tumor necrosis induced by TACE promoted the release of tumor antigens and then had some positive regulatory effects on the anticancer immunity. However, there have been no related studies to testify this hypothesis. Thus, this study aimed to determine the changes in immune function indicated by the peripheral Treg cell proportion in HCC patients after GSMs-TACE. The results of this study provided a piece of preliminary evidence that GSMs-TACE may be used in combination with immune adjuvant therapies to increase the efficacy of HCC treatment.

\section{Patients and Methods}

\subsection{Patients}

Fifty-nine patients with HCC diagnosed in our hospital from July 2016 to June 2019 were enrolled (Table 1). All patients were confirmed as HCC by two or more imaging examinations or liver tumor biopsy, according to the Expert Consensus on Regulation of Standardized Diagnosis and Treatment of Primary Liver Cancer. Twenty-eight patients were followed up regularly after GSMs -TACE treatment for 30 days and the Treg cells proportion in peripheral blood was examined (Fig. 1, Table 2). Ten healthy volunteers were selected as control group.

Table 1 Characteristics of 59 patients with HCC

\begin{tabular}{ll}
\hline Variables & Results \\
\hline $\begin{array}{l}\text { Average age (years, mean } \pm \text { SD) } \\
\text { Gender }\end{array}$ & $54 \pm 11$ \\
Male & 48 \\
Female & 11 \\
Type of hepatitis & \\
Hepatitis B & 51 \\
No hepatitis & 8 \\
Child-Pugh grade & \\
A & 52 \\
B & 7 \\
BCLC stage &
\end{tabular}




\begin{tabular}{|c|c|}
\hline Variables & Results \\
\hline B & 23 \\
\hline $\mathrm{C}$ & 36 \\
\hline \multicolumn{2}{|c|}{$\operatorname{AFP}(n g / m L)$} \\
\hline ¡20 & $18 ? ¿ ?$ \\
\hline 20 & 41 \\
\hline \multicolumn{2}{|c|}{ Tumor size $(\mathrm{cm})$} \\
\hline ¡ 8 & $20 ? \gtreqless ?$ \\
\hline 8 & 39 \\
\hline \multicolumn{2}{|c|}{ Surgical removal of tumors (cases) } \\
\hline Yes & 25 \\
\hline no & 34 \\
\hline \multicolumn{2}{|c|}{ Extrahepatic metastasis (cases) } \\
\hline Yes & 29 \\
\hline no & 30 \\
\hline \multicolumn{2}{|c|}{ Portal or hepatic vein invasion (cases) } \\
\hline Yes & 34 \\
\hline no & 25 \\
\hline \multicolumn{2}{|c|}{ Tumor encapsulation } \\
\hline Yes & 24 \\
\hline no & 35 \\
\hline
\end{tabular}

AFP: alpha-fetoprotein; BCLC: Barcelona Clinic Liver Cancer (BCLC) staging system; HCC, hepatocellular carcinoma

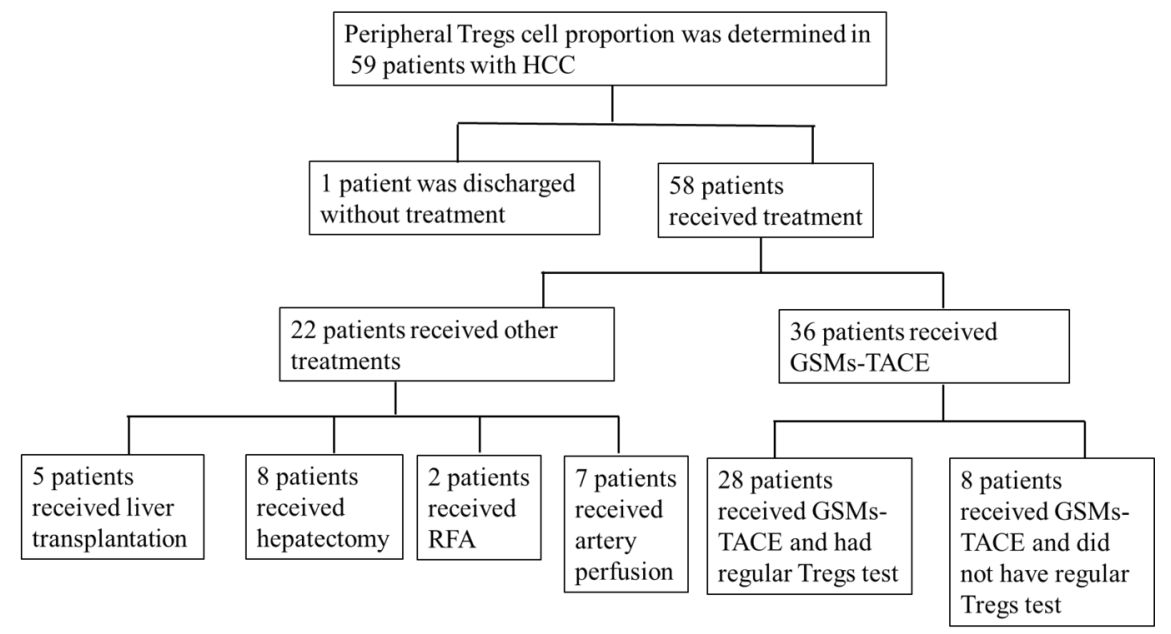

Fig. 1 Flow chart of the enrollment of 28 HCC patients treated with GSMs-TACE. (RFA: radiofrequency ablation; HCC: hepatocellular carcinoma; GSMs-TACE, transarterial chemoembolization with gelatin sponge microparticles)

Table 2 Characteristics of 28 HCC patients who received GSMs-TACE

\begin{tabular}{ll}
\hline Variables & Results \\
\hline Average age (years, mean \pm SD) & $54 \pm 11$
\end{tabular}




\begin{tabular}{ll}
\hline Variables & Results \\
\hline Gender & \\
Male (cases) & 24 \\
Female (cases) & 4 \\
Type of hepatitis & \\
Hepatitis B & 24 \\
None & 4 \\
Child-Pugh grade & \\
A & 25 \\
B & 3 \\
BCLC stage & \\
B & 10 \\
C & 18 \\
AFP(ng/mL) & $? \dot{¿} ?$ \\
20 & 20 \\
i20 & 8 \\
Tumor size (cm) & \\
¡ & $5 ? \dot{\jmath} ?$ \\
Surgical removal of tumor (cases) & 23 \\
Yes & \\
no & 15 \\
Extrahepatic metastasis (cases) & 13 \\
no & \\
Portal or hepatic vein invasion (cases) & 12 \\
Yes & 16 \\
Tumor encapsulation & 17 \\
Yes & 11 \\
Tumor response at 1 month postoperatively & \\
CR & 0 \\
PR & 18 \\
SD & 25 \\
PD & 3 \\
\hline & 0 \\
\hline
\end{tabular}

AFP: alpha-fetoprotein; BCLC: Barcelona Clinic Liver Cancer (BCLC) staging system; HCC: hepatocellular carcinoma; GMPs-TACE, transarterial chemoembolization with gelatin sponge microparticles. CR: complete response; PR: partial response; SD: stable disease; PD: progressive disease.

2.2 The standardized GSMs-TACE

The enrolled patients were treated with GSMs-TACE. The right femoral artery was punctured by Seldinger method and RH hepatic duct was introduced. Celiac arteriography and common hepatic arteriography were performed routinely. All the blood supply arteries of the tumors were identified according to the location, size and staining integrity of the tumors, combined with imaging data, and the auxiliary angiograms of the diaphragm artery, superior mesenteric artery, right renal artery, intercostal artery, left gastric artery, and internal thoracic arteries to determine whether there was a collateral blood supply artery to the tumor. Microparticles of different sizes $(150 \mu \mathrm{m}, 350-560 \mu \mathrm{m}$ and $560-710 \mu \mathrm{m})$ were selected during the procedure according to the tumor size and degree of staining. For patients with large tumors (diameter greater than 5 
$\mathrm{cm}$ ) and abundant nutrient arteries, GSMs of 350 or $560 \mu \mathrm{m}$ in diameter were chosen; for patients with small tumors (diameter less than $5 \mathrm{~cm}$ ) with no abundant blood supply arteries, GSMs of $150 \mu \mathrm{m}$ in diameter were selected as the embolic agent. The appropriate dose of epirubicin (30-50 mg) was decided according to the tumor volume, and the dose of GSMs embolic agent $(50-200 \mathrm{mg}$ ) was selected according to the tumor volume and degree of staining. Epirubicin was diluted with 50-100 $\mathrm{mL}$ of saline and then mixed with embolic agents of GSMs evenly. When the GSMs achieved a uniform and sparse suspension in epirubicin dilution, the mixture was used to slowly embolize the feeding artery of the tumor until the tumor staining disappeared completely, and the embolization usually lasted for 20-30 min.

\subsection{Collection and treatment of blood sample}

All patients signed the informed consent. Functions of liver and kidney, blood test and AFP were routinely examined 1 day before intervention and 4, 10, 20 and 30 days after surgery. Upper abdominal CT plain scan was performed 4 days after operation, and enhanced CT or MRI was performed 30 days after operation. 2 $\mathrm{mL}$ forearm venous blood was collected from all patients with HCC 1 day before operation, 10 days and 30 days after operation using blood collection tube treated with heparin sodium anticoagulant. Flow cytometry was used to determine Treg cell proportion in peripheral blood. $2 \mathrm{ml}$ of forearm venous blood was also collected from the control group for the determination of Treg cell counts in peripheral blood, and the data were collected for statistical analysis.

2.4 Blood preparation and determination of Treg cell proportion by flow cytometry

(1) Venipuncture blood was collected using anticoagulant tube;

(2) $100 \mathrm{~mL}$ whole blood was added to the bottom of the dry powder reagent tube which has antibodies of CD25/CD4/CD127/CD3 in the bottom;

(3) the tube was vortexed for $0.5-1 \mathrm{~s}$ to mix well and incubated for $15 \mathrm{~min}$ at room temperature with light avoided;

(4) $500 \mu \mathrm{L}$ of erythrocyte lysate was added to the tube. The tube was then incubated for 15 min at room temperature with light avoided;

(5) $2 \mathrm{ml}$ PBS solution was added to each tube, and then oscillate the tube. Then the mixture was centrifuged at room temperature for 5 min with a centrifugal force of $300 \mathrm{~g}$;

(6) the supernatant was removed, and then step 5 was repeated for one more time.

(7) the supernatant was removed, and then $500 \mathrm{~mL}$ PBS solution was added to each tube. The sample was then used for flow cytometry within $1 \mathrm{~h}$;

(8) the gating strategy of flow cytometry was set as follows: Treg: cell population of CD4 ${ }^{+}$and CD3+; $\mathrm{CD} 25^{+}$and $\mathrm{CD} 127^{+}$low.

2.5 Statistical analysis

The statistical analysis was conducted by SPSS software (provided by IBM, version 20.0). Results were expressed as mean $\pm \mathrm{SD}$. The $t$ test was used to compare the data of different groups. The test level was $\alpha=0.05$, and $\mathrm{P}<0.05$ was considered statistically significant.

\section{Results}

3.1 An increased proportion of Treg cells in peripheral blood of patients with primary HCC

The percentage of Treg cells $\left(\mathrm{CD} 25^{+}, \mathrm{CD} 127^{+}\right.$Low $)$in Th cells $\left(\mathrm{CD} 3^{+}, \mathrm{CD} 4^{+}\right)$was $11.26 \pm 1.31 \%$ in $\mathrm{HCC}$ patients and $7.32 \pm 1.63 \%$ in healthy control group. The proportion of Treg cells in peripheral blood of HCC patients was significantly higher than that of healthy control group $(\mathrm{P}<0.05)$.

3.2 The association between the peripheral Treg cells proportion and clinical characteristics of HCC 
The peripheral Treg cell proportion in HCC patients was associated with tumor stage, AFP, tumor size and tumor encapsulation. The proportion of Treg cells in peripheral blood of HCC patients at stage $\mathrm{C}$ was higher than that of patients at stage B. The Treg cells proportion in AFP positive $(>20 \mathrm{ng} / \mathrm{mL}) \mathrm{HCC}$ patients was higher than that in AFP negative patients. In addition, the larger the tumor size $(>8 \mathrm{~cm})$, the higher the Treg cell proportion in peripheral blood. According to the imaging findings and DSA tumor staining, the Treg proportion in HCC patients without tumor encapsulation was higher than that in HCC patients with tumor encapsulation $(\mathrm{P}<0.05)$ (Table 3). Analysis showed that metastasis or vascular invasion was not positively correlated with the Treg cell proportion $\left(\mathrm{P}_{\dot{0}} 0.05\right)$.

Table 3 The association between the Treg cell proportion with clinical characteristics of HCC

\begin{tabular}{|c|c|c|c|c|c|}
\hline Characteristics & & Number & $\begin{array}{l}\text { Treg cells } \\
\text { proportion }\end{array}$ & $t$ value & $p$ \\
\hline \multirow{2}{*}{$\begin{array}{l}\text { Tumor stage } \\
\text { (BCLC) }\end{array}$} & stage B & 23 & $10.71 \pm 1.52$ & & \\
\hline & stage $\mathrm{C}$ & 36 & $11.61 \pm 1.03$ & 2.50 & 0.017 \\
\hline \multirow{2}{*}{$\operatorname{AFP}(\mathrm{ng} / \mathrm{mL})$} & $¡ 20$ & 18 & $9.77 \pm 0.99$ & & \\
\hline & {$[?] 20$} & 41 & $11.92 \pm 0.80$ & 8.81 & $¡ 0.001$ \\
\hline \multirow{2}{*}{$\begin{array}{l}\text { Tumor size } \\
(\mathrm{cm})\end{array}$} & ¡8 & 20 & $10.55 \pm 1.37$ & & \\
\hline & {$[?] 8$} & 39 & $11.63 \pm 1.13$ & 3.02 & 0.005 \\
\hline \multirow{2}{*}{$\begin{array}{l}\text { Tumor } \\
\text { encapsulation }\end{array}$} & yes & 35 & $11.77 \pm 1.04$ & & \\
\hline & no & 24 & $10.51 \pm 1.32$ & 4.135 & ¡0.001 \\
\hline Extrahepatic & yes & 29 & $11.11 \pm 1.31$ & 0.887 & 0.379 \\
\hline metastasis & no & 30 & $11.41 \pm 1.31$ & & \\
\hline $\begin{array}{l}\text { Portal or } \\
\text { invasion }\end{array}$ & yes & 34 & $11.60 \pm 1.10$ & 1.932 & 0.058 \\
\hline hepatic vein & no & 25 & $10.95 \pm 1.43$ & & \\
\hline
\end{tabular}

AFP: alpha-fetoprotein; BCLC: Barcelona Clinic Liver Cancer (BCLC) staging system; HCC, hepatocellular carcinoma.

3.3 The Treg cell proportion in peripheral blood of 28 patients with HCC before and at 10 and 30 days after GSMs-TACE

In this study, we observed the Treg cell proportion in HCC patients before operation, at 10 and 30 days after GSMs-TACE, since the normal cycle of interventional therapy was 30 days. Results indicated that the Treg cell proportion after GSMs-TACE showed a slightly decreasing trend at the initial stage of operation (from $11.78 \pm 1.76 \%$ before operation to $8.85 \pm 1.23 \%$ at 10 days after operation), and then a slightly slow uptrend $(10.27 \pm 1.28 \%$ at 30 days after operation), which indicated the necessity of the next cycle of TACE therapy. Compared with before operation, the Treg cell proportion in peripheral blood was statistically significantly lower at 10 and 30 days after operation $(\mathrm{P}<0.05)$ (Table 4, Fig. 2). Moreover, the BCLC subgroup analysis showed that the peripheral Treg proportion of both stage $\mathrm{B}$ and stage $\mathrm{C}$ patients decreased significantly at 10 days after GSMs-TACE compared with before $(\mathrm{P}<0.05)$ (Table 5$)$. The Treg cell proportion in stage $\mathrm{C}$ patients at 30 days after operation was still significantly lower than that before operation. The Treg cell proportion in stage B patients increased at 30 days after TACE compared with 10 days after operation, but was not significantly different from that before TACE $(\mathrm{P}=0.14)$.

Table 4 Comparison of peripheral Treg cell proportions in 28 HCC patients before and after GSMs -TACE. 


\begin{tabular}{llllllll}
$\mathrm{b} 1$ & $\mathrm{~b} 2$ & $\mathrm{~b} 2$ & $\mathrm{~b} 2$ & $\mathrm{~b} 2$ & $\mathrm{~b} 2$ & $\mathrm{~b} 2$ & $\mathrm{~b} 3$ \\
$\mathrm{c} 1$ & $\mathrm{c} 1$ & $\mathrm{c} 1$ & $\mathrm{c} 2$ & $\mathrm{c} 2$ & $\mathrm{c} 3$ & $\mathrm{c} 3$ & $\mathrm{c} 3$ \\
$\mathrm{~d} 1$ & $\mathrm{~d} 1$ & $\mathrm{~d} 1$ & $\mathrm{~d} 2$ & $\mathrm{~d} 2$ & & & \\
$\mathrm{~d} 3$ & $\mathrm{~d} 3$ & $\mathrm{~d} 3$ & $\mathrm{~d} 4$ & $\mathrm{~d} 4$ & & & \\
\hline
\end{tabular}

Fig. 3 (a1) DSA angiography which showed tumor staining during GSMs-TACE in patients with HCC. The feeding artery came from the right hepatic artery. (a2) after GSMs embolization, the blood flow velocity of the feeding artery slowed down. DSA angiography after embolization showed that the tumor staining basically disappeared. (b1) The preoperative abdominal enhanced CT scan of one patient with stage BCLC B before operation showed that the right lobe of the liver was not homogeneously enhanced in the arterial phase. (b2) 4 days after GSMs-TACE, there were honeycomb-like low-density changes in tumors shown on CT plain scan. (b3) abdominal enhanced CT of the same patient 1 month after operation indicated that most of the tumors in the liver were liquefied and necrotic without progression. C. The Treg cell proportion in peripheral blood of one patient before, at 10 and 30 days after GSMs-TACE. (c1) The Treg cell proportion in peripheral blood before GSMs-TACE was 13.28\%; (c2) The Treg cell proportion in peripheral blood at 10 days after GSMs-TACE was 8.56\%; (c3) The Treg cell proportion in peripheral blood at 30 days after GSMs-TACE was $10.60 \%$. (d1) DSA angiography of one patient with liver cancer at BCLC stage B during GSMs-TACE showed that the blood supply artery came from the right hepatic artery. After embolized with gelatin sponge particles, the blood flow velocity of the right hepatic artery slowed down. (d2) DSA angiography indicated that the tumor staining basically disappeared after operation. (d3) the plain abdominal CT of this patient after operation showed that the right hepatic lobe tumor had honeycomb liquefying necrosis and the right portal vein tumor thrombus showed low density necrosis. (d4) enhanced abdominal MRI at 1 month after operation showed that the tumor necrosis area in the right lobe of the liver had no enhancement, and the tumor thrombus in the right branch of the portal vein had necrosis.

3.5 Flow cytometry of Treg cells in peripheral blood of one HCC patient before and after GSMs-TACE

Representative profiles of high CD25 and low CD127 expressions in the peripheral blood of one patient with HCC are shown in Fig.3c. The Treg cells proportion on the $10^{\text {th }}$ day after GSMs-TACE was significantly lower than that of the preoperative level, and the proportion on the $30^{\text {th }}$ day after GSMs-TACE was increased but still lower than that of the preoperative level.

\section{Discussion}

Currently, HCC is still one of the primary causes of cancer deaths, although the survival rates and prognosis of HCC have been greatly improved by liver transplantation and surgical resection [2]. TACE procedures are currently widely used for various cases of HCC, mostly patients at BCLC stage B. Gelatin sponge microparticle (GSM) is an effective embolization agent, and a study reported that when compared with the ethiodized oil which is a permanent embolization agent, GSMs had many advantages, such as a higher tumor response rate at 1 month after treatment, especially in large tumors [13]. Our previous clinical practices have confirmed the safety and efficacy of GSMs-TACE [5, 14, 15]. It was found that 350-560 $\mu \mathrm{m}$ GSMs combined with single chemotherapy drug TACE procedures was effective and safe in treating elderly HCC without surgical resection, and more elderly patients may have better prognosis [5]. GSMs combined with trans-arterial p53-gene-embolization also achieved satisfactory efficacy in treating BCLC stage B HCC as evidenced by favorable survival rates (100\% for both 6 and 12 months) and no significant complications [14]. Therefore, GSMs were adopted in this study, and imaging scan confirmed the efficacy of GSMs-TACE. DSA angiography during the procedure revealed that after GSMs embolization, the blood flow velocity of tumor feeding arteries, slowed down, and the tumor staining basically disappeared. Moreover, 4 days after GSMsTACE, CT plain scan showed some honeycomb-like low-density changes in tumors compared with before 
the procedure. In addition, in a previous study, after analyzing the data of 37 patients who received GSMsTACE for liver metastases after gastrointestinal tumor surgery, it was observed that the treatment produced various degrees of necrosis and shrinkage of lesions, and even 2 patients achieved a complete response [16]. Considering the close relationship between tumor metastasis and the immunosuppressive TME, these findings prompted us to wonder whether tumor necrosis after GSMs-TACE improved the therapeutic efficacy via regulating the immune-mediated elimination of tumor cells.

Regulatory $\mathrm{T}$ (Treg) cells are a subset of $\mathrm{CD}^{+}{ }^{+} \mathrm{T}$ cells in immune system known to function as "immunesuppressor". It is well recognized that Treg cells play an important role in protecting against excessive inflammation and immune response in physiological or some pathological conditions. Furthermore, it has been noticed that Treg cells are involved in liver diseases including HCC [17]. The infiltration of Treg cells into tumor sites can exert a suppressive effect on host anticancer immunity and thereby become an obstacle to curative anticancer therapy $[10,18,19]$. In the current study, we first compared the Treg cell proportion in peripheral blood between HCC patients and healthy volunteers, and it was found that there was an increased proportion of Treg cells in peripheral blood of patients with HCC $(11.26 \pm 1.31 \%$ and $7.32 \pm 1.63 \%$, respectively), which was in accordance with the previous study [19]. Besides, we analyzed the association between the Treg cell proportion and various clinical indicators of HCC, and it was found that the Treg cell proportion in peripheral blood of HCC patients was closely related to tumor stage, AFP content, and tumor diameter, which further confirmed the association between the Treg cell proportion and HCC. However, the mechanism of the association between peripheral Treg cells and HCC pathophysiological characteristics has not been fully elucidated. Shi et al.proposed that Tregs could modify HCC in the way that potentiates the metastasis since it was found that Treg cell proportions in HCC patients were significantly correlated with biomarkers of tumor cell metastasis such as E-cadherin, vimentin and SNAIL, although these findings were based on the analysis of limited cases [20]. In addition, tumor encapsulation was an important clinicopathological marker of HCC invasiveness, and it was observed that the high Treg cell proportion in peripheral blood of HCC patients was significantly correlated with the presence of tumor encapsulation, which was inconsistent with the published literature, where Gao et al. reported that a high Tregs proportion was associated with the absence of tumor encapsulation [21]. Nevertheless, these await further studies.

The 28 patients enrolled in this study received GSMs-TACE, and the imaging techniques confirmed the success of the procedure. To testify our hypothesis that TACE procedures may also have an impact on anti-cancer immunity, the Treg cell proportion in peripheral blood of these 28 HCC patients was determined before and at 10 and 30 days after TACE. It was found that the peripheral Treg cell proportion at 10 days after TACE was significantly lower than that of before the procedure, and the percentage slightly increased 30 days after TACE but was still significantly lower than that of before the procedure. These results indicated that GSMs-TACE had a positive regulatory effect on immune function. The possible reason is that after the effective embolization of tumor feeding artery, the tumor cell necrosis is induced significantly in a short time, and the tumor load is also significantly reduced, which is related to the significant reduction of the immunosuppressive effect on the body. It is well recognized that many tumors bear tumor antigens and can induce T-cell cytotoxic responses [22]. The release of tumor antigens then led to local microenvironment immune response and a subsequent reduction of Treg cells in peripheral blood. Our results showed that the Treg cells proportion in peripheral blood at 10 days after operation was the lowest, which indicated that it may be the optimal time for immunoadjuvant therapy. The improvement of immune function can be maintained within one month, but with the further proliferation of tumor cells, the immune function gradually recovers to the state of inhibition. Therefore, the follow-up treatment including TACE should be carried out subsequently to further reduce the burden of tumors in order to better restore and enhance the body's anti-tumor immunity.

In recent years, tumor immunotherapies have gained promising results. Programmed cell death protein 1 (PD-1), expressed by various activated immune cells, is an immune checkpoint and can protect against autoimmune responses. It has been revealed that in cancer immunity, tumor cells express PD-L1, the ligand of PD-1, and combine with PD-1 in T cells, thereby suppressing $\mathrm{T}$ cell activities and promoting the differentiation of Treg cells [23]. As a result, the anticancer immune responses are inhibited in tumor 
microenvironment. The immunotherapies targeting PD-1/PD-L1 have successfully improved the outcomes of various types of cancer worldwide, including HCC [24]. A pioneering study analyzed the PD-L1 expression in $240 \mathrm{HCC}$ patients who received surgical resection, and it was found that patients who were positive for PD-L1 showed significant shorter disease-free survival or overall survival than those PD-L1 negative patients [25]. Antibodies of PD-1 or PD-L1 in HCC immunology have shown promising efficacies [26]. However, there are still unmet clinical needs considering that many patients show no response. Since one of the important mechanisms of PD-1/PD-L1 pathway is the promotion of Treg cells differentiation, an interesting and promising suggestion was drawn from this study that GSMs-TACE has the potential to be used in combination with immune adjuvant therapies such as PD-1/PD-L1 pathway targeting therapies to increase the efficacy of HCC treatment.

In conclusion, the Treg cell proportion in peripheral blood of HCC patients was higher than that of healthy controls and was closely related to the clinical characteristics of HCC. The GSMs-TACE procedure significantly reduced the peripheral Treg cell proportions at 10 and 30 days after operation. These results indicated that GSMs-TACE could reduce the proportion of Treg cells in peripheral blood after operation and exert a positive regulatory effect on the immune function of HCC patients. This study provides a piece of preliminary evidence that GSMs-TACE has the potential to be used in combination with immune adjuvant therapies such as therapies targeting PD-1 or PDL-1 to increase the efficacy of HCC treatment.

However, there are also some limitations of this study, and the expression of other molecules or biomarkers involved in tumor immunity should be assessed along with the Treg cell proportion to better explain the mechanism of GSMs-TACE procedures affecting tumor immunity, so as to provide references for the treatment design of HCC patients.

\section{Authors' contributions}

YUEWEI ZHANG and ZHIZHONG REN were responsible for reviewing the concept design, ZHIZHONG REN wrote and proofread the article. YUANXUN YUE created the figures and XIN LIN made important comments on the revision of the article.XUEQIANG ZHAO,YING LIU,XIAOWEI YANG,XIN HUANG,YU ZHENG,ZHANQI WEI participated in literature collection, analysis and summary. YUEWEI ZHANG was responsible for project guidance. All authors read and agree to the final text.

\section{Ethics approval and consent to participate}

Not applicable.

\section{Patient consent for publication}

Not applicable.

\section{Competing interests}

The authors declare that they have no competing interests.

\section{References}

1. Kulik, L. and H.B. El-Serag, Epidemiology and Management of Hepatocellular Carcinoma. Gastroenterology, 2019. 156 (2): p. 477-491 e1.

2. Schlachterman, A., et al., Current and future treatments for hepatocellular carcinoma. World Journal of Gastroenterology, 2015.21 (28): p. 8478-8491.

3. Bruix, J., G.J. Gores, and V. Mazzaferro, Hepatocellular carcinoma: clinical frontiers and perspectives. Gut, 2014.63 (5): p. 844-855.

4. Raoul, J.L., et al., Updated use of TACE for hepatocellular carcinoma treatment: How and when to use it based on clinical evidence. Cancer Treat Rev, 2019. 72 : p. 28-36. 
5. Zhao, G.S., et al., 350-560 mum gelatin sponge particles combined with transcatheter arterial chemoembolization for the treatment of elderly hepatocellular carcinoma: The safety and efficacy. Medicine (Baltimore), 2017. 96 (16): p. e6629.

6. Liu, S., et al., Complete remission of diffuse hepatocellular carcinoma in a young adult after GSP-TACE: a case report. World J Surg Oncol, 2014. 12 : p. 300.

7. Ohue, Y. and H. Nishikawa, Regulatory T (Treg) cells in cancer: Can Treg cells be a new therapeutic target? Cancer Sci, 2019.110 (7): p. 2080-2089.

8. Nishikawa, H. and S. Sakaguchi, Regulatory T cells in tumor immunity. International Journal of Cancer, 2010. 127 (4): p. 759-767.

9. Tanaka, A. and S. Sakaguchi, Targeting Treg cells in cancer immunotherapy. Eur J Immunol, 2019. 49 (8): p. 1140-1146.

10. Curiel, T.J., et al., Specific recruitment of regulatory $T$ cells in ovarian carcinoma fosters immune privilege and predicts reduced survival. Nat Med, 2004. 10 (9): p. 942-9.

11. Burrel, M., et al., Survival of patients with hepatocellular carcinoma treated by transarterial chemoembolisation (TACE) using Drug Eluting Beads. Implications for clinical practice and trial design.Journal of Hepatology, 2012. 56 (6): p. 1330-1335.

12. Meyer, T., et al., Sorafenib in combination with transarterial chemoembolisation in patients with unresectable hepatocellular carcinoma (TACE 2): a randomised placebo-controlled, double-blind, phase 3 trial.Lancet Gastroenterology \& Hepatology, 2017. 2 (8): p. 565-575.

13. Xia, J.L., et al., Study of severe and rare complications of transarterial chemoembolization (TACE) for liver cancer. European Journal of Radiology, 2006. 59 (3): p. 407-412.

14. Liu, Y., et al., Trans-arterial p53-gene-embolization with gelatin sponge microparticles for hepatocellular carcinoma with BCLC stage B: single-center experience. Cell Biochem Biophys, 2015.71 (1): p. 99-104.

15. Zhou, J., et al., Transarterial chemoembolization with gelatin sponge microparticles for barcelona clinic liver cancer Stage $C$ and large hepatocellular carcinoma: Initial clinical experience. J Cancer Res Ther, 2017. 13 (5): p. 767-772.

16. Li, C., et al., A retrospective analysis of the efficacy of microparticle-mediated chemoembolization in liver metastases arising from gastrointestinal tumors. J Cancer Res Ther, 2017. 13 (4): p. 642-646.

17. Zhang, H.R., Z.Z. Jiang, and L.Y. Zhang, Dual effect of T helper cell 17 (Th17) and regulatory T cell (Treg) in liver pathological process: From occurrence to end stage of disease. International Immunopharmacology, 2019. 69 : p. 50-59.

18. Yang, X.H., et al., Increase of CD4(+) CD25(+) regulatory T-cells in the liver of patients with hepatocellular carcinoma. Journal of Hepatology, 2006. 45 (2): p. 254-262.

19. Kiniwa, Y., et al., CD8+ Foxp3+ regulatory T cells mediate immunosuppression in prostate cancer. Clin Cancer Res, 2007.13 (23): p. 6947-58.

20. Shi, C., et al., CD4(+) CD25(+) regulatory $T$ cells promote hepatocellular carcinoma invasion via TGF-beta1-induced epithelial-mesenchymal transition. Onco Targets Ther, 2019.12 : p. 279-289.

21. Gao, Q., et al., Intratumoral balance of regulatory and cytotoxic $T$ cells is associated with prognosis of hepatocellular carcinoma after resection. Journal of Clinical Oncology, 2007.25 (18): p. 2586-2593.

22. Morse, M.A., et al., Immunoregulatory T cells in cancer immunotherapy. Expert Opin Biol Ther, 2002. 2 (8): p. 827-34. 
23. Salmaninejad, A., et al., PD-1/PD-L1 pathway: Basic biology and role in cancer immunotherapy. J Cell Physiol, 2019.234 (10): p. 16824-16837.

24. Seidel, J.A., A. Otsuka, and K. Kabashima, Anti-PD-1 and Anti-CTLA-4 Therapies in Cancer: Mechanisms of Action, Efficacy, and Limitations. Front Oncol, 2018. 8 : p. 86.

25. Gao, Q., et al., Overexpression of PD-L1 significantly associates with tumor aggressiveness and postoperative recurrence in human hepatocellular carcinoma. Clin Cancer Res, 2009. 15 (3): p. 971-9.

26. Hato, T., et al., Immune checkpoint blockade in hepatocellular carcinoma: current progress and future directions. Hepatology, 2014.60 (5): p. 1776-82. 